\title{
Textures and the shapes of domains in Langmuir monolayers
}

\author{
Kok-Kiong Loh and Joseph Rudnick \\ Department of Physics, UCLA \\ 405 Hilgard Ave., Los Angeles, California 90095-1547
}

(November 11, 2018)

\begin{abstract}
Two-dimensional domains containing an $X Y$-like order parameter exhibit non-trivial internal structure and take on shapes controlled by the configuration that the order parameter adopts. The textures exhibited by the order parameter in such domains are controlled by the interplay between bulk and surface contributions to the energy. We report calculations of the internal texture and the shape of such domains. These calculations lead to the determination of the equilibrium properties of two-dimensional domains, such as those observed in Langmuir monolayers. This allows for the unambiguous exploration of the implications of experimental findings.
\end{abstract}

68.55.-a, 68.18.+p, 68.55.Ln, 68.60.-p

Monolayers of surfactants confined to the air/water interface have been found to possess complex textures similar to those observed in liquid crystals. The textures are generally observed in "tilted" phases - that is, in phases in which the long axes of the molecules in the film are not perpendicular to the water surface but are uniformly tilted with respect to the normal. The textures are the result of the spontaneous organization of the molecular tilt azimuth on macroscopic length scales. They can be understood, at least qualitatively, in terms of continuum elastic theories of smectic liquid crystals [1] or, more relevant to the present work, in terms of the two-dimensional $X Y$ model.

When the surfactants organize into domains of condensed tilted phases, such as the $L_{2}$ phase, surrounded by an isotropic phase [2], many striking textures have been observed. Such textures range from continuous boojums, textures with point and/or line defects [1], to striped textures in spiral domains [3]. We will, however, focus our discussions on the boojums in which the tilt azimuth varies continuously and appears to radiate in some cases from a defect located at the edge of the domain or from a "virtual" defect in the isotropic phase [4]. Domains containing a boojum texture are not circular. Rather, they reflect the various nonisotropic influences that control domain shape in the presence of a nonhomogeneous background. Among the features seen are protrusions, at times sharp enough to be characterized as "cusps" [5], and indentations, which give the domains a heartlike, or "cardioid" appearance [6:7].

While there are notable cases in which the full analytical solution can be obtained [5, the determination of the properties of a domain in which such a texture has formed presents a calculational challenge. Energy minimization entails the simultaneous adjustment of the orientations of surfactant molecules in the interior of the domain and the shape of the domain's boundary. Past work on the problem of the shape of surfactant domains has relied on either the assumption of a texture that is unaffected by variations in the boundary [8], or on the approximation of a nearly circular domain [5,9]. Neither assumption is necessarily close to reality, and results obtained in both cases can be legitimately called into question.

This Letter describes a successful evaluation of equilibrium properties of a domain of surfactants. The evaluation is based on the numerical solution of the extremum equations for the energy of an $X Y$-like texture confined to a compact, but not necessarily circular, domain. We are able to explore the texture and the associated domain shape that result from various forms of the boundary and bulk energy of the two-dimensional system.

The starting point in the analysis is the Hamiltonian of the $X Y$ order parameter, represented as the twodimensional unit vector $\hat{c}(x, y)$, which is parameterized in terms of the angle, $\Theta(x, y)$ between $\hat{c}(x, y)$ and the $x$ axis. The order parameter field $\Theta(x, y)$ will be referred to as the texture. The Hamiltonian, $H[\Theta]=$ $\int_{\Omega} \mathcal{H}_{b} d A+\oint_{\Gamma} \sigma(\vartheta-\Theta) d s$, where $\mathcal{H}_{b}$ is given by

$$
\begin{array}{r}
\mathcal{H}_{b}=\frac{\kappa}{2}\left\{|\nabla \Theta|^{2}+\beta\left[\left(-\Theta_{x}^{2}+\Theta_{y}^{2}\right) \cos 2 \Theta\right.\right. \\
\left.\left.-2 \Theta_{x} \Theta_{y} \sin 2 \Theta\right]\right\},
\end{array}
$$

$\Theta_{x}$ and $\Theta_{y}$ represent partial derivatives of $\Theta$ with respect to $x$ and $y$, respectively. The integral $\int_{\Omega}$ is over the domain's bulk, while $\oint_{\Gamma}$ is an integral over the closed curve bounding the domain. The coefficient $\kappa$ is the "mean" Frank constant, the average of the bend and splay moduli, while $\beta$ is proportional to the difference between the two moduli. Specifically, $2 \kappa=K_{s}+K_{b}$ and $2 \kappa \beta=K_{s}-K_{b}$ where $K_{b} / 2$ multiplies $|\vec{\nabla} \times \hat{c}|^{2}$ in the energy of the $X Y$ order parameter and $K_{s} / 2$ multiplies $|\vec{\nabla} \cdot \hat{c}|^{2}$. The constants $K_{b}$ and $K_{s}$ are, respectively, the bend and splay modulus. The boundary energy, $\sigma(\vartheta-\Theta)$, will have the general form

$$
\sigma(\varphi)=a_{0}+a_{1} \cos \varphi+a_{2} \cos 2 \varphi+\cdots
$$

The angle $\vartheta$ in the argument of the boundary energy is the angle between the unit normal to the curve bounding the domain and the $x$ axis. The fact that the harmonic expansion, (2), of the boundary energy consists entirely 
of cosine terms reflects the absence of "chiral" interactions between the texture and the domain boundary.

The minimization of the energy leads to equations for the texture $\Theta(x, y)$ and the bounding curve $\Gamma . \Theta(x, y)$ satisfies both a bulk and a boundary extremum equation. The bulk equation is

$$
\begin{aligned}
-\nabla^{2} \Theta & +\beta\left[\left(\Theta_{x x}-\Theta_{y y}\right) \cos 2 \Theta+2 \Theta_{x y} \sin 2 \Theta\right. \\
& \left.+\left(-\Theta_{x}^{2}+\Theta_{y}^{2}\right) \sin 2 \Theta+2 \Theta_{x} \Theta_{y} \cos 2 \Theta\right]=0 .
\end{aligned}
$$

The double-subscripted $\Theta$ 's represent second partial derivatives with respect to the relevant variables. The extremum equation for $\Theta(x, y)$ on the boundary is

$$
\begin{gathered}
\kappa \Theta_{n}[1-\beta \cos 2(\vartheta-\Theta)] \\
+\kappa \beta \Theta_{t} \sin 2(\vartheta-\Theta)-\sigma^{\prime}(\vartheta-\Theta)=0,
\end{gathered}
$$

where $\Theta_{n}$ and $\Theta_{t}$ are respectively the normal and tangential derivatives. The extremum equation for the bounding curve $\Gamma$ is

$$
\begin{aligned}
& \mathcal{H}_{b}-\sigma^{\prime}(\vartheta-\Theta) \Theta_{n}-\sigma^{\prime \prime}(\vartheta-\Theta) \Theta_{t} \\
& +\left[\sigma(\vartheta-\Theta)+\sigma^{\prime \prime}(\vartheta-\Theta)\right] \frac{d \vartheta}{d s}+\lambda=0 .
\end{aligned}
$$

The quantity $\lambda$ is a Lagrange multiplier that enforces the condition of constant enclosed area.

We first solve numerically for the equilibrium $\Theta(x, y)$ using a variational formulation of the finite element method [10], assuming an initial boundary $\Gamma^{(0)}$. A mesh of triangles is generated over $\Omega$ using an adaptive method that refines the grids where necessary. Functions are defined by their values on the vertices of the triangles. The value of a function elsewhere is obtained by interpolation. The system energy $H[\Theta]$ is then a function of the values of $\Theta(x, y)$ at the vertices, $\Theta \equiv\left(\Theta_{i}\right)$, which can be determined by solving $\partial H(\boldsymbol{\Theta}) / \partial \Theta_{i}=0$ [11], where $i$ runs from 1 to the number of vertices. We label the texture determined at this point $\boldsymbol{\Theta}^{(0)}$.

Equation (5), after being cast into a coordinatedependent form, turns out to be a second order differential equation for $\Gamma$. Substituting $\boldsymbol{\Theta}^{(0)}$ for the $\Theta(x, y)$ in the equation, $\Gamma$ can be determined by the Runge Kutta method [11]. The solution, which is labeled $\Gamma^{(1)}$, is in turn utilized to determine a new texture $\boldsymbol{\Theta}^{(1)}$. The process is iterated until self-consistency is achieved.

Making use of the numerical scheme outlined above, we have investigated the the texture and shape of the bounding curve under the influence of variations in the boundary energy coefficient $a_{2}$ in Eq. (2) and the stiffness coefficient $\beta$. Before presenting our results, we note that when $a_{2}=0$ and $\beta=0$, the exact result [5] is given by a circular boundary of radius $R$, with the boojum texture, i.e., a defect with winding number +2 [8] located at a distance $R_{B} \equiv R\left(1+\sqrt{1+\rho^{2}}\right) / \rho$ from the center of the domain, where $\rho \equiv R a_{1} / \kappa$ is the normalized domain radius. The normalized domain radius $\rho$ will be used as
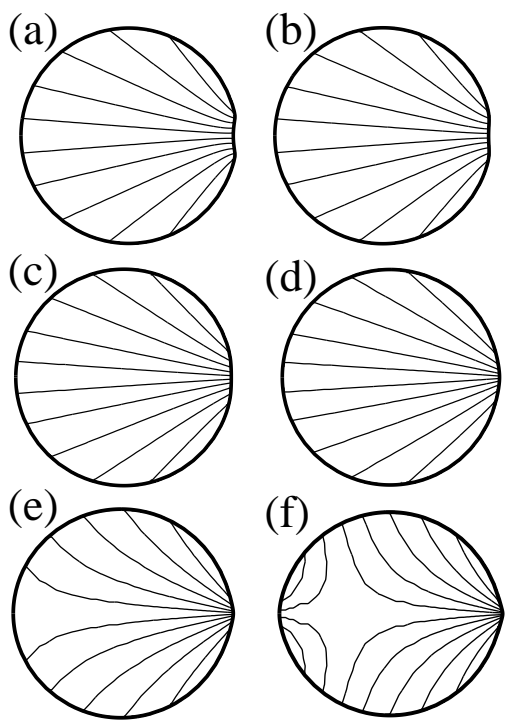

FIG. 1. The constant-order-parameter contours and shapes of domains computed for $-0.5<a_{2}<0.5, \rho=5, a_{0}=4$, $a_{1}=1$. (a) $a_{2}=-0.5$, (b) $a_{2}=-0.3$, (c) $a_{2}=-0.1,($ d) $a_{2}=0.1,(\mathrm{e}) a_{2}=0.3$ and (f) $a_{2}=0.5$. (a)

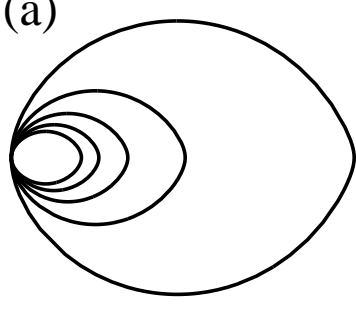

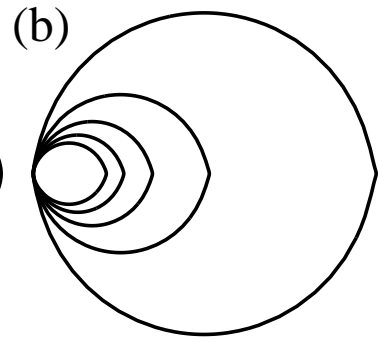

FIG. 2. The shapes of domains of various sizes computed for $a_{0}=4, a_{1}=1$ and $a_{2}=0.6$. (a)Smaller domains with $\rho=0.2,0.25,0.33,0.5,1$ which exhibit 2 -fold symmetry. (b)Larger domains with $\rho=2,2.5,3.3,5,10$ which have a protrusion on one end of the boundary. Each of the sets of domains are plotted to scale.

a gauge of the domain size throughout the discussion. For cases in which the domains are not circular, $R$ is the effective radius, in that $\pi R^{2}$ will be the area of the domain.

We first look at our result by varying only $a_{2}$ while keeping $\beta=0$. In the limit of very small domains, $\rho \ll 1$, the line tension anisotropy has very little effect on the texture, which exhibits almost no spatial variation. The boundary response of the domain is not affected by the $a_{1}$ contribution. If $a_{2} \neq 0$, small domains exhibit twofold symmetry. They become elongated when $a_{2}>0$, and they flatten at both ends when $a_{2}<0$. For larger domains in which $\rho \sim 1$ and $a_{2} / a_{1} \ll 1$, we find a protrusion when $a_{2}>0$, and an indentation when $a_{2}<0$. The feature lies on the end of the domain boundary closest to the virtual defect. The loss of twofold symmetry is largely due to the fact the dominant contribution of 

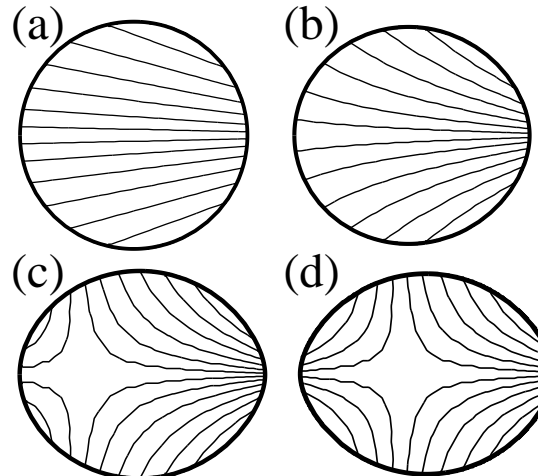

(d)
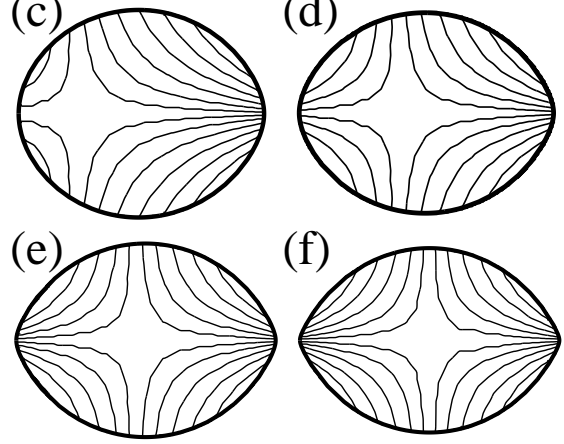

(f)

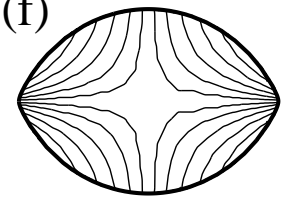

FIG. 3. The constant-order-parameter contours and the shapes of domains with $\rho=1$ and $a_{0}=4$. The coefficients of the anisotropic line-tension are (a) $a_{1}=1, a_{2}=0$, (b) $a_{1}=0.8, a_{2}=0.2,(\mathrm{c}) a_{1}=0.6, a_{2}=0.4,(\mathrm{~d}) a_{1}=0.4$, $a_{2}=0.6,(\mathrm{e}) a_{1}=0.2, a_{2}=0.8$ and (f) $a_{1}=0, a_{2}=1$.

the domain texture, which in turn induces deformation through $a_{2}$, comes from the $a_{1}$ contribution in the boundary anisotropy. Boundary features are more prominent as the domain size increases. Figure 1 shows the shapes of domains for $-0.5<a_{2}<0.5$ for $\rho=5$. Numerically, the boundary features are well-defined for domains of sizes $\rho \sim 1$. To the numerical accuracy that we are able to achieve, there is no discontinuity in slope on the domain boundary. That this ought to be the case can be verified analytically 12,13]. The numerical result we have is compatible with those discussed in Refs. [5,8, 12]. For even larger domains, $\rho \gg 1$, the features are confined in a small portion of the boundary. The domains become nearly circular again in the large- $\rho$ limit. Up to the largest domain we have examined, $\rho=32$, we can identify protrusions when $a_{2} / a_{0}>0.1$. Because of the rapid texture variation in the immediate vicinity of the boundary, associated with the approach to the boundary of the virtual boojum singularity, we are unable to perform dependable numerical investigations of extremely large domains. This leaves open the question of the asymptotic behavior of the domain in the large- $\rho$ regime. Figure 2 shows the shapes of domains in which the normalized radius ranges from $\rho=0.2$ to $\rho=10$ for $a_{1}=1$ and $a_{2}=0.6$.

As regards the texture, we are able to numerically reproduce the exact result of Rudnick and Bruinsma [5] when $a_{1}=0$ and $a_{2}=1$ and the boundary is fixed at a circle. This texture is associated with two virtual defects. When the boundary is allowed to relax, the domain acquires a "cigar shape". When both $a_{1}$ and $a_{2}$ are not
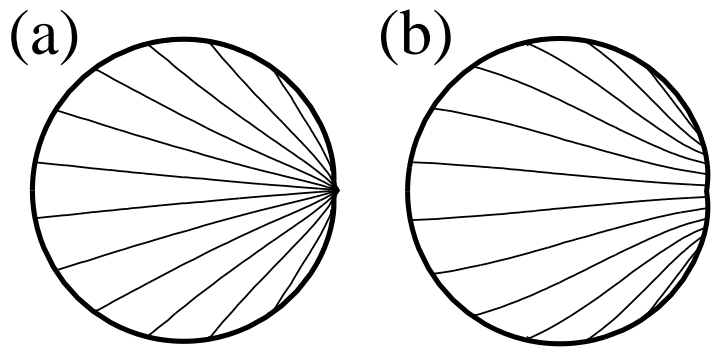

FIG. 4. The constant-order-parameter contours and the shapes of domains with $\rho=8, a_{0}=4, a_{1}=1.6$ and $a_{2}=0$. Their stiffness coefficients are (a) $\beta=-0.8$, (b) $\beta=0.8$.
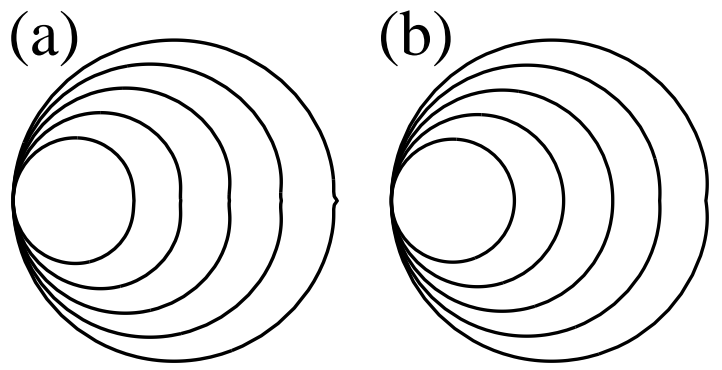

FIG. 5. The domain shapes computed for $a_{0}=4, a_{1}=1.6$ and $a_{2}=0$, and $\rho=0.5,1,2,4,8$. Their stiffness coefficients are (a) $\beta=-0.8$, (b) $\beta=0.8$. For ease of observation, domains are not shown to scale.

equal to zero, the texture can be thought of as a superposition of pure $a_{1}$ and pure $a_{2}$ textures. Typically for $\rho \sim 1$, the effect of the second defect becomes observable, in the form of a distortion of constant-order-parameter contours, when $a_{2} / a_{1} \approx 1 / 4$. The progressive changes of the texture and domain shape from a system with pure $a_{1}$ to a system with pure $a_{2}$ are depicted in Fig. 3. Domains with indentations, protrusions, and cigar-shaped domains, have all been observed [7].

We now investigate the textures and boundaries that result when $\beta \neq 0$. The parameters $a_{0}$ and $a_{1}$ are assumed to be finite, while all other $a_{i}$ 's are set equal to zero. We have computed the textures and domain shapes for $-0.8<\beta<0.8$ and $0.5<\rho<8$. When $|\beta| \leq 0.5$, our results are in qualitative agreement with those reported in Ref. [8]. For $\beta<0$, the texture is modified as if the virtual singularity has moved closer to the domain boundary, and the domain acquires a very small protruding feature. On the other hand, when $\beta>0$, the texture relaxes as if the virtual defect has moved away from the boundary, and the boundary correction is similar to that of a domain with an indentation. There are, however, quantitative differences. The textures obtained numerically deviate significantly from the boojum textures utilized in Refs. [8, 14]. The sizes of the features on the boundaries are no more than $1 \%$ of the overall domain radii. We do not expect such features to be observable experimentally.

When $|\beta|=0.8$ and $\rho=8$, the domains are no longer 
circular in appearance. Domains acquire small protrusions for negative $\beta$. Domains have an indentation when $\beta$ is positive. Figure 1 shows the domain shapes and textures computed with $\beta= \pm 0.8$ for $\rho=8$. The deviation from the boojum texture is more clearly observable when $\beta=+0.8$ in Fig. 四b. The constant-order-parameter contours distort so as to push the virtual defect away from the boundary. Figure 5 shows the changes in the domain shape when $\rho$ decreases from 8 to 0.5 for $\beta= \pm 0.8$. The amplitude of the protrusion of the domain with negative $\beta$ decreases with $\rho$. At the same time, segments of the boundary with negative curvature start to develop adjacent to the protrusion. At $\rho=2$, the negative curvature segments of the boundary overwhelm the protrusion and give the domain an indentation. When $\rho=0.5$, the indentation disappears, while the protrusion continues to decrease in amplitude. The domain appears circular. The complex boundary behavior described above can be attributed to the rapid variation of the order parameter along the boundary near the singularity. In contrast to this behavior, the size of the indentation on the boundary decreases monotonically as $\rho$ decreases when $\beta=0.8$. The domain appears circular at $\rho \leq 2$. It is the strength of the splay modulus that smooths out the variation of the order parameter along the segment of the boundary near the singularity and results in simple boundary behavior.

Our attempts to compare our results with experiments indicate that all the observed domain shapes in Refs. [7.9.14] can be accounted for, a least qualitatively, by line-tension anisotropy alone. Although we have established the existence of nontrivial domain shapes due to purely elastic anisotropy, domains with the protrusions and indentations discussed immediately above occur only when the anisotropy is very strong $|\beta|=0.8$. Furthermore, the protrusions that are generated in our calculations do not resemble those reported to have been observed experimentally 9.14. We conclude that linetension anisotropy must be present in such monolayers. On the other hand, both line-tension and elastic anisotropies may be responsible for indentations on the boundary of domains of the compressed phase. Further measurements on the size dependence of the domain shapes are needed to identify the underlying mechanism.

We note here that our analysis is based on a highly simplified model of monolayers. Other factors that contribute to the boundary shapes and textures - such as the dipolar interaction [15], the tilt degree of freedom [16] and the bond-orientational ordering [1] - have been neglected. However, the results reported here are useful in that they do indicate the influence of various features that make up the model discussed.

In conclusion, we have devised a numerical scheme that enables us to solve simultaneously for the minimum energy configuration of an $X Y$ order parameter confined to a two-dimensional domain and the extremal shape of the boundary of that domain. We have utilized the method to investigate the response of the texture-boundary system under the influence of a nontrivial anisotropy in the boundary energy as well as the bulk elastic energy. Both line-tension anisotropy and elastic anisotropy result in nontrivial domain shapes. The observed domains with protrusions and cigar shape can be attributed to the linetension anisotropy. On the other hand, elastic anisotropy cannot be ruled out as the source of the observed cardioids.

We are grateful to Professor Charles Knobler, Professor Robijn Bruinsma and Dr. Jiyu Fang for very useful discussions.

[1] T.M. Fischer, R.F. Bruinsma and C.M. Knobler, Phys. Rev. E 50, 413 (1994).

[2] For a description of monolayer phases see C. M. Knobler and R.C. Desai, Ann. Rev. Phys. Chem. 43, 207 (1992).

[3] R.M. Weis and H.M. McConnell, J. Phys. Chem. 894453 (1985).

[4] S. A. Langer and J. P. Sethna, Phys. Rev. A 34, 5035 (1986). For the original discussion of boojums, see N. D. Mermin in Quantum Fluids and Solids, S. B. Trickey, E. Adams and J. Duffy, eds. (Plenum, New York, 1977).

[5] J. Rudnick and R. Bruinsma, Phys. Rev. Lett. 74, 2491 (1994).

[6] G. Brezesinski, E. Scalas, B. Struth, H. Möhwald, F. Bringezu, U. Gehlert, G. Weidemann and D. Vollhardt, J. Phys. Chem. 99, 8758 (1995).

[7] J. Fang and C.M. Knobler, unpublished.

[8] P. Galatola and J.B. Fournier, Phys. Rev. Lett. 75, 3297 (1994).

[9] J. Fang, E. Teer, C.M. Knobler, K.-K. Loh and J. Rudnick, Phys. Rev. E 56, 1859(1997).

[10] C. Johnson, Numerical solution of partial differential equations by the finite element method (Cambridge University Press, Cambridge, 1987).

[11] W.H. Press, B.P. Flannery, S.A. Teukolsky and W.T. Vetterling, Numerical Recipes in C, The Art of Scientific Computing (Cambridge University Press, Cambridge, 1991).

[12] K.-K. Loh and J. Rudnick, unpublished.

[13] In light of this result, the claims in [5] of a cusp-like feature in the form of a discontinuity in the slope of the bounding curve cannot be true. In fact, the result is the consequence of an error in the analysis reported in that paper.

[14] S. Rivière and J. Meunier, Phys. Rev. Lett. 74, 2495 (1995).

[15] M.A. Mayer and T.K. Vanderlick, J. Chem. Phys. 100, 8399 (1994).

[16] Y. Tabe, N. Shen, E. Mazur and H. Yokoyama, unpublished. 